\title{
Three attractively interacting fermions in a harmonic trap: Exact solution, ferromagnetism, and high-temperature thermodynamics
}

\author{
Xia-Ji Liu, ${ }^{*}$ Hui $\mathrm{Hu},{ }^{\dagger}$ and Peter D. Drummond ${ }^{\ddagger}$ \\ ARC Centre of Excellence for Quantum-Atom Optics, Centre for Atom Optics and Ultrafast Spectroscopy, \\ Swinburne University of Technology, Melbourne 3122, Australia
}

(Received 16 June 2010; published 30 August 2010)

\begin{abstract}
Three fermions with strongly repulsive interactions in a spherical harmonic trap constitute the simplest nontrivial system that can exhibit the onset of itinerant ferromagnetism. Here, we present exact solutions for three trapped, attractively interacting fermions near a Feshbach resonance. We analyze energy levels on the upper branch of the resonance where the atomic interaction is effectively repulsive. When the $s$-wave scattering length $a$ is sufficiently positive, three fully polarized fermions are energetically stable against a single spin-flip, indicating the possibility of itinerant ferromagnetism, as inferred in the recent experiment. We also investigate the high-temperature thermodynamics of a strongly repulsive or attractive Fermi gas using a quantum virial expansion. The second and third virial coefficients are calculated. The resulting equations of state can be tested in future quantitative experimental measurements at high temperatures and can provide a useful benchmark for quantum Monte Carlo simulations.
\end{abstract}

DOI: $10.1103 /$ PhysRevA.82.023619

PACS number(s): 03.75.Ss, 03.75.Hh, 05.30.Fk

\section{INTRODUCTION}

Few-particle systems have become increasingly crucial to the physics of strongly interacting ultracold quantum gases [1-3]. Because of large interaction parameters, conventional perturbation theory approaches to quantum gases, such as mean-field theory, simply break down [2-4]. A small ensemble of a few fermions and/or bosons, which is either exactly solvable or numerically tractable, is more amenable to nonperturbative quantal calculations. Although challenging experimentally, such ensembles benefit from the same unprecedented controllability and tunability as in a mesoscopic system containing a hundred thousand particles. The atomic species, the quantum statistics, the $s$-wave and higher partial-wave interactions [5], and the external trapping environment can all be controlled experimentally. The study of few-particle systems can therefore give valuable insights into the more complicated mesoscopic many-body physics of a strongly interacting quantum gas. In addition to qualitative insights, these solutions have already proved invaluable in developing high-temperature quantum virial or cluster expansions for larger systems [6], which have been recently verified experimentally [7].

The purpose of this article is to add a further milestone in this direction. By exactly solving the eigenfunctions of three attractively interacting fermions in a spherical harmonic trap, we aim to give a few-body perspective of itinerant ferromagnetism in an effectively repulsive Fermi gas, which was observed as a transient phenomenon in a recent measurement at MIT [8]. This is possible because the quantum three-body problem with $s$-wave interactions is exactly soluble in three dimensions. It is interesting to recall that the corresponding classical three-body problem is notoriously insoluble. The

\footnotetext{
*xiajiliu@swin.edu.au

†hhu@swin.edu.au

†pdrummond@swin.edu.au
}

reason for this unexpectedly docile quantum behavior is that the $s$-wave interaction Hamiltonian applicable to ultracold Bose and Fermi gases is essentially just a boundary condition on an otherwise noninteracting quantum gas. Thus, we have an unusual situation where quantum mechanics actually simplifies an intractable classical problem.

For Bose-Einstein condensates (BEC) in the strongly interacting regime, three trapped bosonic atoms with large $s$-wave scattering length were already investigated theoretically as a minimum prototype [9] of this few-body physics. To understand the fascinating crossover from a BEC to a BCS superfluid, two spin-up and two spin-down fermions in a trap were also simulated numerically, constituting the simplest model of the BEC-BCS crossover problem [10,11]. Moreover, knowledge of few-particle processes such as threebody recombination is primarily responsible for controlling the loss rate or lifetime of ultracold atomic gases, which, in many cases, imposes severe limitations on experiments. Important examples in this context include the confirmation of stability of dimers in the BEC-BCS crossover [12] and the discovery of the celebrated Efimov state (i.e., a bound state of three resonantly interacting bosons) as well as the related universal four-body bound state [13].

Whether an itinerant Fermi gas with repulsive interactions exhibits ferromagnetism is a long-standing problem in condensed matter physics [14]. It has recently attracted increasing attention in the cold-atom community [15-24]. The answer depends on a competition between the repulsive interaction energy and the cost of kinetic energy arising from Pauli exclusion. A strong repulsive interaction can induce polarization or ferromagnetism, since fermions with the same spin orientation are protected from local interactions by the exclusion principle. This, however, increases the Fermi energy, as all fermions must now occupy the same band. The difficulty in finding the transition point is that quantum correlations change the interaction energy in a way that is difficult to calculate in general. 
As early as the 1930s, Stoner [14] showed with a simple model using mean-field theory that ferromagnetism in a homogeneous Fermi gas will always take place. This model, however, gives the unphysical result that the interaction energy within the mean-field approximation scales linearly with the $s$-wave scattering length $a$ and therefore could be infinitely large. The predicted critical interaction strength at zero temperature, $\left(k_{F} a\right)_{c}=\pi / 2$, where $k_{F}$ is the Fermi wave-vector is also too large in the mean-field picture. An improved prediction from second-order perturbation theory, $\left(k_{F} a\right)_{c} \simeq$ 1.054 , suffers from similar doubts about its validity $[15,18]$. Most recently, three independent ab initio quantum Monte Carlo simulations conclusively reported a zero-temperature ferromagnetic phase transition at $\left(k_{F} a\right)_{c} \simeq 0.8-0.9[19,22,23]$.

Several important issues are still open, including the nature of transition at finite temperatures. The unitarity limited interaction energy at infinitely large scattering length $(a \rightarrow \infty)$ is also to be determined.

The exciting experiment at MIT of ${ }^{6} \mathrm{Li}$ atoms realized in some sense the textbook Stoner model [8]. A crucial aspect in this realization is that the interatomic interactions are very different from the conventional model of hard-sphere interactions $[15,22,23]$. In the experiment, the atoms are on the upper branch of a Feshbach resonance with a positive scattering length $a>0$ and a negligible effective interaction range. The properties of the atoms are therefore universal, independent of the details of the interactions [25,26]. This universality, however, comes at a price: the underlying two-body interaction is always attractive, so that the ground state is a gas of molecules of size $a$. The experiment thus suffers from considerable atom loss and has to be carried out under nonequilibirum conditions. This is clearly explained in Fig. 1, which shows the relative energy spectrum of a pair of fermions in a harmonic trap with frequency $\omega$ across a Feshbach resonance [27]. For $a>0$, the whole spectrum consists of two distinct parts, the lowest ground-state branch diverging as $E_{\text {rel }} \simeq-\hbar^{2} /\left(m a^{2}\right)$ and the regular upper branch having a finite energy. In the context of this two-body picture, an $s$-wave "repulsive" Fermi gas is realized, provided that there are no pairs of fermions occupying the ground state branch of molecules. However, as far as the many-particle aspect is concerned, it is not clear to what extent this two-body picture of a repulsive Fermi gas will persist. In other words, can we prove rigorously that the whole Hilbert space of an attractive many-fermion system with a positive scattering length consists exactly of a sub-Hilbert space of a repulsive Fermi gas with the same scattering length, together with an orthogonalized subspace of molecules?

This article addresses the problem of itinerant ferromagnetism in an attractive Fermi gas using a few-particle perspective by examining the exact solutions for the energy spectrum of three trapped, attractively interacting fermions in their upper branch of the Feshbach resonance. Our main results may be summarized as follows.

(1) First, we present an elegant and physically transparent way to exactly solve the Hamiltonian of three interacting fermions in a harmonic trap. The method may easily be generalized to treat other systems with different types of atomic species, geometries, and interactions.

(2) Second, we observe clearly from the whole relative energy spectrum of three attractive fermions (see Fig. 3) that

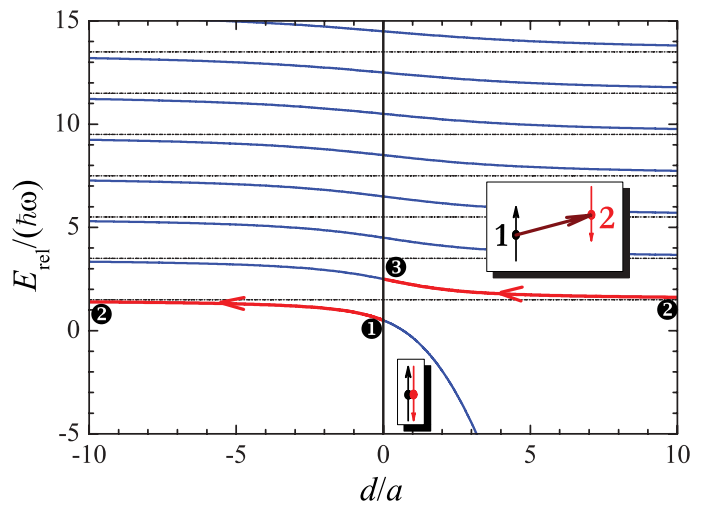

FIG. 1. (Color online) Energy spectrum of the relative motion of a trapped two-fermion system near a Feshbach resonance (i.e, $d / a=0$, where $d$ is the characteristic harmonic oscillator length). For a positive scattering length $a>0$ in the right part of the figure, the ground state is a molecule with size $a$, whose energy diverges as $E_{\mathrm{rel}} \simeq-\hbar^{2} /\left(m a^{2}\right)$. The excited states or the upper branch of the resonance may be viewed as the Hilbert space of a repulsive Fermi gas with the same scattering length $a$. In this two-body picture, the level from the point 2 to 3 is the ground-state energy level of the repulsive two-fermion subspace, whose energy initially increases linearly with increasing $a$ from $1.5 \hbar \omega$ at the point 2 and finally saturates toward $2.5 \hbar \omega$ at the resonance point 3. For comparison, we illustrate as well the ground-state energy level in the case of a negative scattering length and show how the energy increases with increased scattering length from point 1 to 2 .

there are indeed two branches of the spectrum on the side of positive scattering length. As the scattering length goes to an infinitely small positive value, the lower branch diverges in energy to $-\infty$, while another upper branch always converges to the noninteracting limit. The latter may be interpreted as the energy spectrum of three "repulsively" interacting fermions. However, close to the Feshbach resonance, there are many nontrivial avoided crossings between two types of spectra, making it difficult to unambiguously identify a repulsive Fermi system. These avoided crossings are expected in more general cases and lead to nontrivial consequences in a time-dependent field-sweep experiment passing from the weakly interacting regime at $a=0^{+}$to the unitarity limit at $a=+\infty$.

(3) Third, we show exactly that near the Feshbach resonance, three repulsively' interacting fermions in a spherical harmonic trap, say, in a two spin-up and one spin-down configuration, are higher in total energy than three fully polarized fermions [see the ground-state energy in Fig. 3(b)]. Thus, there must be a ferromagnetic transition occurring at a certain critical scattering length. Note that ferromagnetism cannot be obtained in a two-fermion system. As shown in Fig. 1, even at resonance the total ground-state energy of a repulsively interacting pair, $E_{\text {pair }}=4 \hbar \omega$, which is the sum of the relative ground-state energy $E_{\text {rel }}=2.5 \hbar \omega$ and the zero-point energy of center-of-mass motion $E_{\mathrm{cm}}=1.5 \hbar \omega$, cannot be larger than the total ground-state energy of two fully polarized fermions, that is, $E_{\uparrow \uparrow}=4 \hbar \omega$.

(4) Last but most importantly, we obtain the hightemperature equations of state of strongly interacting Fermi gases (see Figs. 4, 5, 6, and 7), within a quantum virial expansion theory, which was developed recently by the 
present authors [6]. The second and third virial (expansion) coefficients of both attractive and repulsive Fermi gases can be calculated, using the full energy spectrum of three interacting fermions. In the unitarity limit, we find that in the high-temperature regime where our quantum virial expansion is reliable, the itinerant ferromagnetism disappears.

The article is organized as follows. In the next section, we outline the theoretical model for a few fermions with $s$-wave interactions in a spherical harmonic trap. In Sec. III, we explain how to construct the exact wave functions for three interacting fermions and discuss in detail the whole energy spectrum. In Sec. IV we develop a quantum virial expansion for thermodynamics and calculate the second and third virial coefficients, based on the full energy spectrum of two-fermion and three-fermion systems, respectively. The high-temperature equations of state of strongly interacting Fermi gases are then calculated and discussed in Sec. V. Finally, Sec. VI is devoted to conclusions and some final remarks. The appendices show the numerical details of the exact solutions.

\section{MODELS}

Consider a few fermions in a three-dimensional (3D) isotropic harmonic trap $V(\mathbf{x})=m \omega^{2} x^{2} / 2$ with the same mass $m$ and trapping frequency $\omega$, occupying two different hyperfine states or two spin states. The fermions with unlike spins attract each other via a short-range $s$-wave contact interaction. It is convenient to use the Bethe-Peierls boundary condition to replace the $s$-wave pseudopotential. That is, when any particles $i$ and $j$ with unlike spins are close to each other, $r_{i j}=\mid \mathbf{x}_{i}-$ $\mathbf{x}_{j} \mid \rightarrow 0$, the few-particle wave function $\psi\left(\mathbf{x}_{1}, \mathbf{x}_{2}, \ldots, \mathbf{x}_{N}\right)$ with proper symmetry should satisfy [28-30]

$$
\psi=\mathcal{A}_{i j}\left(\mathbf{X}_{i j}=\frac{\mathbf{x}_{i}+\mathbf{x}_{j}}{2},\left\{\mathbf{x}_{k \neq i, j}\right\}\right)\left(\frac{1}{r_{i j}}-\frac{1}{a}\right),
$$

where $\mathcal{A}_{i j}\left(\mathbf{X}_{i j},\left\{\mathbf{x}_{k \neq i, j}\right\}\right)$ is a function independent of $r_{i j}$, and $a$ is an $s$-wave scattering length. This boundary condition can be equivalently written as

$$
\lim _{r_{i j} \rightarrow 0} \frac{\partial\left(r_{i j} \psi\right)}{\partial r_{i j}}=-\frac{r_{i j} \psi}{a} .
$$

Otherwise, the wave function $\psi$ obeys a noninteracting Schrödinger equation:

$$
\sum_{i=1}^{N}\left[-\frac{\hbar^{2}}{2 m} \nabla_{\mathbf{x}_{i}}^{2}+\frac{1}{2} m \omega^{2} x_{i}^{2}\right] \psi=E \psi
$$

We now describe how to solve all the wave functions with energy level $E$ for a two- or three-fermion system.

\section{METHOD}

In a harmonic trap, it is useful to separate the center-of-mass motion and the relative motion. We thus define the following center-of-mass coordinate $\mathbf{R}$ and relative coordinates $\mathbf{r}_{i}(i \geqslant 2)$ for $N$ fermions in a harmonic trap $[29,30]$ as

$$
\mathbf{R}=\left(\mathbf{x}_{1}+\cdots+\mathbf{x}_{N}\right) / N
$$

and

$$
\mathbf{r}_{i}=\sqrt{\frac{i-1}{i}}\left(\mathbf{x}_{i}-\frac{1}{i-1} \sum_{k=1}^{i-1} \mathbf{x}_{k}\right)
$$

respectively. In this Jacobi coordinate, the Hamiltonian of the noninteracting Schrödinger equation takes the form $\mathcal{H}_{0}=$ $\mathcal{H}_{\mathrm{cm}}+\mathcal{H}_{\text {rel }}$, where

$$
\mathcal{H}_{\mathrm{cm}}=-\frac{\hbar^{2}}{2 M} \nabla_{\mathbf{R}}^{2}+\frac{1}{2} M \omega^{2} R^{2}
$$

and

$$
\mathcal{H}_{\mathrm{rel}}=\sum_{i=2}^{N}\left[-\frac{\hbar^{2}}{2 m} \nabla_{\mathbf{r}_{i}}^{2}+\frac{1}{2} m \omega^{2} r_{i}^{2}\right]
$$

The center-of-mass motion is simply that of a harmonically trapped particle of mass $M=N m$, with well-known wave functions and spectrum $E_{\mathrm{cm}}=\left(n_{\mathrm{cm}}+3 / 2\right) \hbar \omega$, where $n_{\mathrm{cm}}=$ $0,1,2, \ldots$, is a non-negative integer. In the presence of interactions, the relative Hamiltonian should be solved in conjunction with the Bethe-Peierls boundary condition, Eq. (2).

\section{A. Two fermions in a 3D harmonic trap}

Let us first briefly revisit the two-fermion problem in a harmonic trap, where the relative Schrödinger equation becomes

$$
\left[-\frac{\hbar^{2}}{2 \mu} \nabla_{\mathbf{r}}^{2}+\frac{1}{2} \mu \omega^{2} r^{2}\right] \psi_{2 b}^{\mathrm{rel}}(\mathbf{r})=E_{\mathrm{rel}} \psi_{2 b}^{\mathrm{rel}}(\mathbf{r}),
$$

where two fermions with unlike spins do not stay at the same position $(r>0)$. Here, we have redefined $\mathbf{r}=\sqrt{2} \mathbf{r}_{2}$ and have used a reduced mass $\mu=m / 2$. It is clear that only the $l=0$ subspace of the relative wave function is affected by the $s$-wave contact interaction. According to the Bethe-Peierls boundary condition, as $r \rightarrow 0$ the relative wave function should take the form $\psi_{2 b}^{\mathrm{rel}}(r) \rightarrow(1 / r-1 / a)$ or satisfy $\partial\left(r \psi_{2 b}^{\mathrm{rel}}\right) / \partial r=$ $-\left(r \psi_{2 b}^{\mathrm{rel}}\right) / a$. The two-fermion problem in a harmonic trap was first solved by Busch and co-workers [27]. Here, we present a simple physical interpretation of the solution.

The key point is that, regardless of the boundary condition, there are two types of general solutions of the relative Schrödinger equation, Eq. (8), in the $l=0$ subspace, $\psi_{2 b}^{\text {rel }}(r) \propto$ $\exp \left(-r^{2} / 2 d^{2}\right) f(r / d)$. Here the function $f(x)$ can either be the first kind of Kummer confluent hypergeometric function ${ }_{1} F_{1}$ or the second kind of Kummer confluent hypergeometric function $U$. We have taken $d=\sqrt{\hbar / \mu \omega}$ as the characteristic length scale of the trap. In the absence of interactions, the first Kummer function gives rise to the standard wave function of 3D harmonic oscillators. With interactions, however, we have to choose the second Kummer function $U$, since it diverges as $1 / r$ at the origin and thus satisfies the Bethe-Peierls boundary condition.

Therefore, the (unnormalized) relative wave function and relative energy should be rewritten as

$$
\psi_{2 b}^{\mathrm{rel}}(r ; v)=\Gamma(-v) U\left(-v, \frac{3}{2}, \frac{r^{2}}{d^{2}}\right) \exp \left(-\frac{r^{2}}{2 d^{2}}\right)
$$


and

$$
E_{\mathrm{rel}}=\left(2 v+\frac{3}{2}\right) \hbar \omega
$$

respectively. Here, $\Gamma$ is the Gamma function, the real number $v$ plays the role of a quantum number and should be determined by the boundary condition $\lim _{r \rightarrow 0} \partial\left(r \psi_{2 b}^{\mathrm{rel}}\right) / \partial r=-\left(r \psi_{2 b}^{\mathrm{rel}}\right) / a$. By examining the short-range behavior of the second Kummer function $U(-v, 3 / 2, x)$, this leads to the familiar equation for energy levels,

$$
\frac{2 \Gamma(-v)}{\Gamma(-v-1 / 2)}=\frac{d}{a} .
$$

In Fig. 1, we give the resulting energy spectrum as a function of the dimensionless interaction strength $d / a$.

The spectrum is easy to understand. At infinitely small scattering length $a \rightarrow 0^{-}, v\left(a=0^{-}\right)=n_{\text {rel }}\left(n_{\text {rel }}=0,1,2, \ldots,\right)$, which recovers the spectrum in the noninteracting limit. With increasingly attractive interactions, the energies decrease. In the unitarity (resonance) limit where the scattering length diverges, $a \rightarrow \pm \infty$, we find that $v(a= \pm \infty)=n_{\text {rel }}-1 / 2$. As the attraction increases further, the scattering length becomes positive and decreases in magnitude. We then observe two distinct types of behavior: the ground state is a molecule of size $a$, whose energy diverges asymptotically as $-\hbar^{2} / m a^{2}$ as $a \rightarrow 0^{+}$, while the excited states may be viewed as two repulsively interacting fermions with the same scattering length $a$. Their energies decrease to the noninteracting values as $a \rightarrow 0^{+}$.

In this two-body picture, a universal repulsively interacting Fermi gas with zero-range interaction potentials may be realized on the positive scattering length side of a Feshbach resonance for an attractive interaction potential, provided that all two fermions with unlike spins occupy the excited states or the upper branch of the two-body energy spectrum.

\section{B. Three fermions in a 3D harmonic trap}

Let us turn to the three-fermion case by considering two spin-up fermions and one spin-down fermion, that is, the $\uparrow \downarrow \uparrow$ configuration shown in Fig. 2. The relative Hamiltonian can be written as $[29,30]$

$$
\mathcal{H}_{\text {rel }}=\frac{\hbar^{2}}{2 \mu}\left(\nabla_{\mathbf{r}}^{2}+\nabla_{\rho}^{2}\right)+\frac{1}{2} \mu \omega^{2}\left(r^{2}+\rho^{2}\right),
$$

where we have redefined the Jacobi coordinates $\mathbf{r}=\sqrt{2} r_{2}$ and $\rho=\sqrt{2} r_{3}$, which measure the distance between the particles 1 and 2 (i.e., pair) and the distance from the particle 3 to the center-of-mass of the pair, respectively.

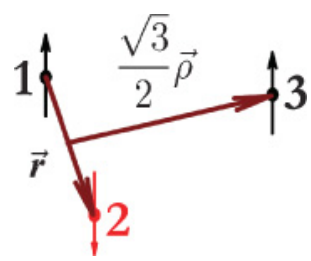

FIG. 2. (Color online) Configuration of three interacting fermions, two spin-up and one spin-down.

\section{General exact solutions}

Inspired by the two-fermion solution, it is readily seen that the relative wave function of the Hamiltonian (12) may be expanded into products of two Kummer confluent hypergeometric functions. Intuitively, we may write down the following ansatz [6],

$$
\psi_{3 b}^{\mathrm{rel}}(\mathbf{r}, \rho)=\left(1-\mathcal{P}_{13}\right) \chi(\mathbf{r}, \rho),
$$

where

$$
\chi(\mathbf{r}, \rho)=\sum_{n} a_{n} \psi_{2 b}^{\mathrm{rel}}\left(r ; v_{l, n}\right) R_{n l}(\rho) Y_{l}^{m}(\hat{\rho}) .
$$

The two-body relative wave function $\psi_{2 b}^{\mathrm{rel}}\left(r ; v_{l, n}\right)$ with energy $\left(2 v_{l, n}+3 / 2\right) \hbar \omega$ describes the motion of the paired particles 1 and 2 , and the wave function $R_{n l}(\rho) Y_{l}^{m}(\hat{\rho})$ with energy $(2 n+l+3 / 2) \hbar \omega$ gives the motion of particle 3 relative to the pair. Here, $R_{n l}(\rho)$ is the standard radial wave function of a 3D harmonic oscillator and $Y_{l}^{m}(\hat{\rho})$ is the spherical harmonic. Owing to the rotational symmetry of the relative Hamiltonian (12), it is easy to see that the relative angular momenta $l$ and $m$ are good quantum numbers. The value of $v_{l, n}$ is uniquely determined from energy conservation,

$$
E_{\text {rel }}=\left[\left(2 v_{l, n}+3 / 2\right)+(2 n+l+3 / 2)\right] \hbar \omega,
$$

for a given relative energy $E_{\text {rel }}$. It varies with the index $n$ at a given angular momentum $l$. Finally, $\mathcal{P}_{13}$ is an exchange operator for particles 1 and 3 , which ensures the correct exchange symmetry of the relative wave function due to the Fermi exclusion principle, that is, $\mathcal{P}_{13} \chi(\mathbf{r}, \rho)=$ $\chi(\mathbf{r} / 2+\sqrt{3} \rho / 2, \sqrt{\mathbf{3}} \mathbf{r} / 2-\rho / 2)$. The relative energy $E_{\text {rel }}$ together with the expansion coefficient $a_{n}$ should be determined by the Bethe-Peierls boundary condition, that is, $\lim _{r \rightarrow 0}\left[\partial r \psi_{3 b}^{\text {rel }}(\mathbf{r}, \rho)\right] / \partial r=-\left[r \psi_{3 b}^{\text {rel }}(\mathbf{r}, \rho)\right] / a$. We note that the second Bethe-Peierls boundary condition in the case of particle 2 approaching particle 3 is satisfied automatically due to the exchange operator acting on the relative wave function.

By writing $\chi(\mathbf{r}, \rho)=\phi(r, \rho) Y_{l}^{m}(\hat{\rho})$, the Bethe-Peierls boundary condition takes the form $(r \rightarrow 0)$

$$
-\frac{1}{a}[r \phi(r, \rho)]=\frac{\partial[r \phi(r, \rho)]}{\partial r}-(-1)^{l} \phi\left(\frac{\sqrt{3} \rho}{2}, \frac{\rho}{2}\right) .
$$

Using the asymptotic behavior of the second kind of Kummer function, $\lim _{x \rightarrow 0} \Gamma\left(-v_{l, n}\right) U\left(-v_{l, n}, 3 / 2, x^{2}\right)=\sqrt{\pi} / x-$ $2 \sqrt{\pi} \Gamma\left(-v_{l, n}\right) / \Gamma\left(-v_{l, n}-1 / 2\right)$, it is easy to show that, in the limit of $r \rightarrow 0$,

$$
-\frac{1}{a}[r \phi(r, \rho)]=-\frac{\sqrt{\pi}}{a} \sum_{n} a_{n} R_{n l}(\rho)
$$

and

$$
\frac{\partial[r \phi(r, \rho)]}{\partial r}=-\sqrt{\pi} \sum_{n} a_{n} R_{n l}(\rho) \frac{2 \Gamma\left(-v_{l, n}\right)}{\Gamma\left(-v_{l, n}-1 / 2\right)} .
$$

Thus, the Bethe-Peierls boundary condition becomes

$$
\sum_{n} a_{n}\left[B_{n} R_{n l}(\rho)-R_{n l}\left(\frac{\rho}{2}\right) \psi_{2 b}^{\mathrm{rel}}\left(\frac{\sqrt{3} \rho}{2} ; v_{l, n}\right)\right]=0
$$


where

$$
B_{n}=(-1)^{l} \sqrt{\pi}\left[\frac{d}{a}-\frac{2 \Gamma\left(-v_{l, n}\right)}{\Gamma\left(-v_{l, n}-1 / 2\right)}\right] .
$$

Projecting onto the orthogonal and complete set of basis functions $R_{n l}(\rho)$, we find a secular equation,

$$
\frac{2 \Gamma\left(-v_{l, n}\right)}{\Gamma\left(-v_{l, n}-1 / 2\right)} a_{n}+\frac{(-1)^{l}}{\sqrt{\pi}} \sum_{n^{\prime}} C_{n n^{\prime}} a_{n^{\prime}}=\left(\frac{d}{a}\right) a_{n},
$$

where we have defined the matrix coefficient,

$$
C_{n n^{\prime}} \equiv \int_{0}^{\infty} \rho^{2} d \rho R_{n l}(\rho) R_{n^{\prime} l}\left(\frac{\rho}{2}\right) \psi_{2 b}^{\mathrm{rel}}\left(\frac{\sqrt{3} \rho}{2} ; v_{l, n^{\prime}}\right),
$$

which arises from the exchange effect due to the operator $\mathcal{P}_{13}$. In the absence of $C_{n n^{\prime}}$, the aforementione secular equation describes a three-fermion problem of a pair and a single particle, uncorrelated to each other. It then simply reduces to Eq. (11), as expected.

The secular equation, Eq. (21), was first obtained by Kestner and Duan [31] by solving the three-particle scattering problem using the Green function. To solve it, for a given scattering length we may try different values of relative energy $E_{\text {rel }}$, implicit via $v_{l, n}$. However, it turns out to be more convenient to diagonalize the matrix $\mathbf{A}=\left\{A_{n n^{\prime}}\right\}$ for a given relative energy, where

$$
A_{n n^{\prime}}=\frac{2 \Gamma\left(-v_{l, n}\right)}{\Gamma\left(-v_{l, n}-1 / 2\right)} \delta_{n n^{\prime}}+\frac{(-1)^{l}}{\sqrt{\pi}} C_{n n^{\prime}} .
$$

The eigenvalues of the matrix $\mathbf{A}$ then gives all the possible values of $d / a$ for a particular relative energy. We finally invert $a\left(E_{\text {rel }}\right)$ to obtain the relative energy as a function of the scattering length. Numerically, we find that the matrix $\mathbf{A}$ is symmetric and thus the standard diagonalization algorithm can be used. We outline the details of the numerical calculation of Eq. (23) in Appendix A.

\section{Exact solutions in the unitarity limit}

In the unitarity limit with infinitely large scattering length, $a \rightarrow \infty$, we may obtain more physical solutions using hyperspherical coordinates, as shown by Werner and Castin $[28,30]$. By defining a hyperradius $R=\sqrt{\left(r^{2}+\rho^{2}\right) / 2}$ and hyperangles $\vec{\Omega}=(\alpha, \hat{r}, \hat{\rho})$, where $\alpha=\arctan (r / \rho)$ and $\hat{r}$ and $\hat{\rho}$ are, respectively, the unit vector along $\mathbf{r}$ and $\rho$, we may write $[28,30]$

$$
\psi_{3 b}^{\mathrm{rel}}(R, \vec{\Omega})=\frac{F(R)}{R}\left(1-\mathcal{P}_{13}\right) \frac{\varphi(\alpha)}{\sin (2 \alpha)} Y_{l}^{m}(\hat{\rho})
$$

to decouple the motion in the hyperradius and the hyperangles for given relative angular momenta $l$ and $m$. This leads to the following decoupled Schrödinger equations [30]:

$$
-F^{\prime \prime}-\frac{1}{R} F^{\prime}+\left(\frac{s_{l, n}^{2}}{R^{2}}+\omega^{2} R^{2}\right) F=2 E_{\mathrm{rel}} F
$$

and

$$
-\varphi^{\prime \prime}(\alpha)+\frac{l(l+1)}{\cos ^{2} \alpha} \varphi(\alpha)=s_{l, n}^{2} \varphi(\alpha),
$$

where $s_{l, n}^{2}$ is the eigenvalue for the $n$th wave function of the hyperangle equation.
For three fermions, $s_{l, n}^{2}$ is always positive. Therefore, the hyperradius equation (25) can be interpreted as a Schrödinger equation for a fictitious particle of mass unity moving in two dimensions in an effective potential $\left(s_{l, n}^{2} / R^{2}+\omega^{2} R^{2}\right)$ with a bounded wave function $F(R)$. The resulting spectrum is $[28,30]$

$$
E_{\mathrm{rel}}=\left(2 q+s_{l, n}+1\right) \hbar \omega,
$$

where the good quantum number $q$ labels the number of nodes in the hyperradius wave function.

The eigenvalue $s_{l, n}$ should be determined by the BethePeierls boundary condition, which in hyperspherical coordinates takes the form [28,30]

$$
\varphi^{\prime}(0)-(-1)^{l} \frac{4}{\sqrt{3}} \varphi\left(\frac{\pi}{3}\right)=0 .
$$

In addition, we need to impose the boundary condition $\varphi(\pi / 2)=0$, since the relative wave function (24) should not be singular at $\alpha=\pi / 2$. The general solution of the hyperangle equation, Eq. (26), satisfying $\varphi(\pi / 2)=0$ is given by

$$
\varphi \propto x^{l+1}{ }_{2} F_{1}\left(\frac{l+1-s_{l, n}}{2}, \frac{l+1+s_{l, n}}{2}, l+\frac{3}{2} ; x^{2}\right),
$$

where $x=\cos (\alpha)$ and ${ }_{2} F_{1}$ is the hypergeometric function. In the absence of interactions, the Bethe-Peierls boundary condition (28) should be replaced by $\varphi(0)=0$, since the relative wave function (24) should not be singular at $\alpha=0$ either. As $\varphi(0)=\Gamma(l+3 / 2) \Gamma(1 / 2) /\left[\Gamma\left(\left(l+2+s_{l, n}\right) / 2\right) \Gamma((l+2-\right.$ $\left.\left.\left.s_{l, n}\right) / 2\right)\right]$, this boundary condition leads to $\left[l+2-s_{l, n}^{(1)}\right] / 2=$ $-n$, or $s_{l, n}^{(1)}=2 n+l+2$, where $n=0,1,2, \ldots$, is a nonnegative integer and we have used the superscript " 1 " to denote a noninteracting system. However, a spurious solution occurs when $l=0$ and $n=0$, for which $s_{l, n}^{(1)}=2, \varphi(\alpha)=\sin (2 \alpha) / 2$ and, thus, the symmetry operator $\left(1-\mathcal{P}_{13}\right)$ gives a vanishing relative wave function in Eq. (24) that should be discarded [30]. We conclude that, for three noninteracting fermions,

$$
s_{l, n}^{(1)}= \begin{cases}2 n+4, & l=0, \\ 2 n+l+2, & l>0 .\end{cases}
$$

For three interacting fermions, we need to determine $s_{l, n}$ by substituting the general solution (29) into the Bethe-Peierls boundary condition (28). In Appendix B, we describe how to accurately calculate $s_{l, n}$. In the boundary condition, Eq. (28), the leading effect of interactions is carried by $\varphi^{\prime}(0)$ and, therefore, $\varphi^{\prime}(0)=0$ determines the asymptotic values of $s_{l, n}$ at large momentum $l$ or $n$. This gives rise to $\left(l+1-\bar{s}_{l, n}\right) / 2=$ $-n$, or

$$
\bar{s}_{l, n}= \begin{cases}2 n+3, & l=0, \\ 2 n+l+1, & l>0,\end{cases}
$$

where we have used a bar to indicate the asymptotic results. By comparing Eqs. (30) and (31), asymptotically the attractive interaction will reduce $s_{l, n}$ by a unity.

\section{Energy spectrum of three interacting fermions}

We have numerically solved both the general exact solution (13) along the BEC-BCS crossover and the exact solution (24) in the unitarity limit. In the latter unitary case, the accuracy of 

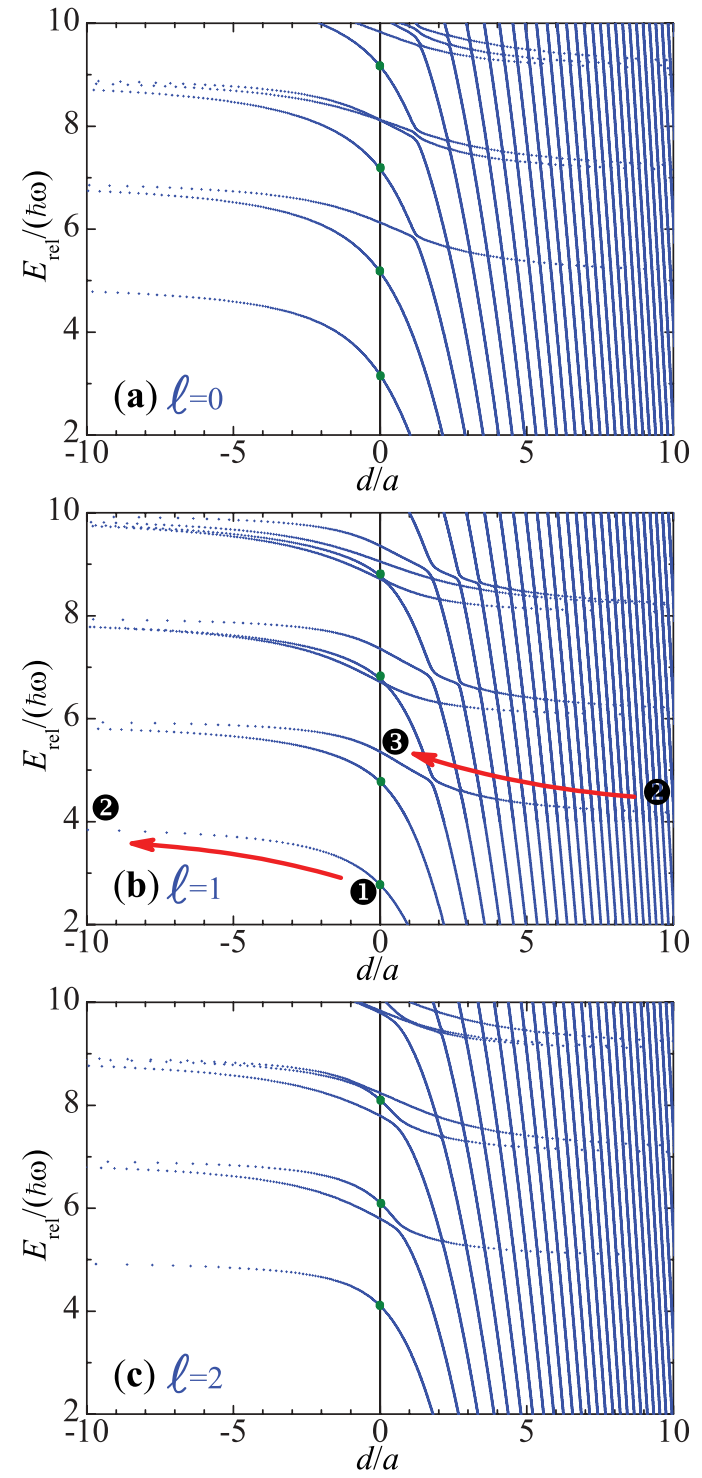

FIG. 3. (Color online) Relative energy spectrum of three interacting fermions at different subspaces or relative angular momenta $l$. On the positive scattering length (BEC) side of the resonance, there are two types of energy levels: one (is vertical and) diverges with decreasing the scattering length $a$ and the other (is horizontal) converges to the noninteracting spectrum. The latter may be viewed as the energy spectrum of three repulsively interacting fermions. In analogy with the two-fermion case, we show in the ground-state subspace $(l=1)$ the ground-state energy level of the repulsive three-fermion system (i.e, from point 2 to point 3 ), as well as the ground-state energy level of the attractive three-fermion system for $a<0$ (i.e., from the point 1 to point 2). In the unitarity limit, we show by the circles the energy levels that should be excluded when we identify the energy spectrum for infinitely large repulsive interactions.

results can be improved to arbitrary precision by using suitable mathematical software, described in Appendix B. Figure 3 reports the energy spectrum of three interacting fermions with increasingly attractive interaction strength at three total relative angular momenta, $l=0,1$, and 2 . For a given scattering length, we typically calculate several ten thousand energy levels [i.e., $\left.E_{\text {rel }}<(l+256) \hbar \omega\right]$ in each different subspace.
To construct the matrix A, Eq. (23), we have kept a maximum value of $n_{\max }=128$ in the functions $R_{n l}(\rho)$. Using the accurate spectrum in the unitarity limit as a benchmark, we estimate that the typical relative numerical error of energy levels is less than $10^{-6}$. We have found a number of nontrivial features in the energy spectrum.

The spectrum on the BCS side is relatively simple. It can be understood as a noninteracting spectrum at $d / a \rightarrow-\infty$, in which $E_{\mathrm{rel}}=(2 Q+3) \hbar \omega$ at $l=0$ and $E_{\mathrm{rel}}=(2 Q+l+$ $1) \hbar \omega$ at $l \geqslant 1$, with a positive integer $Q=1,2,3, \ldots$, that denotes also the degeneracy of the energy levels. The attractive interactions reduce the energies and at the same time lift the degeneracy. Above the resonance or unitary point of $d / a=0$, however, the spectrum becomes much more complicated.

There are a group of nearly vertical energy levels that diverge toward the BEC limit of $d / a \rightarrow+\infty$. From the two-body relative energy spectrum in Fig. 1, we may identify these as energy states containing a molecule of size $a$ and a fermion. For a given scattering length, these nearly vertical energy levels differ by about $2 \hbar \omega$, resulting from the motion of the fermion relative to the molecule. In addition to the nearly vertical energy levels, most interestingly, we observe also some nearly horizontal energy levels, which converge to the noninteracting spectrum in the BEC limit. In analogy with the two-body case, we may identify these horizontal levels as the energy spectrum of three repulsively interacting fermions. We show explicitly in Fig. 3(b) the ground-state level of three repulsively interacting fermions, which increases in energy from point 2 to point 3 with increasing scattering length from $a=0^{+}$to $a=+\infty$. For comparison, we also show the ground-state level of three attractively interacting fermions at a negative scattering length, which decreases in energy from point 2 to point 1 with increasing absolute value of $a$.

This identification of energy spectrum for repulsive interactions, however, is not as rigorous as in the two-body case. There are many apparent avoided crossings between the vertical and horizontal energy levels. Therefore, by changing a positive scattering length from the BEC limit to the unitarity limit, three fermions initially at the horizontal level may finally transition to a vertical level, provided that the sweep of scattering length is sufficiently slow and adiabatic. This leads to the conversion of fermionic atoms to bosonic molecules. A detailed analysis of the loss rate of fermionic atoms as a function of sweep rate may be straightforwardly obtained by applying the Landau-Zener tunneling model.

Let us now focus on the resonance case of most significant interest. In Fig. 3, we show explicitly by green (solid) dots the vertical energy levels in the unitarity limit. These levels should be excluded if we are interested in the spectrum of repulsively interacting fermions. Amazingly, for each given angular momentum, these energy levels form a regular ladder with an exact energy spacing of $2 \hbar \omega$ [29]. Using the exact solution in the unitarity limit, Eq. (27), we may identify unambiguously that the energy ladder is given by

$$
E_{\mathrm{rel}}=\left(2 q+s_{l, 0}+1\right) \hbar \omega .
$$

Therefore, in the unitarity limit the lowest-order solution of the hyperangle equation gives rise to the relative wave function 
of a molecule and a fermion. Thus, it should be discarded when considering three resonantly interacting fermions with an effective repulsive interaction.

This observation immediately leads to the ground-state energy of three repulsively interacting fermions,

$$
E_{\mathrm{gs}}^{\uparrow \downarrow \uparrow}=\left(s_{1,1}+1\right) \hbar \omega+1.5 \hbar \omega \simeq 6.858249309 \hbar \omega,
$$

including the zero-point energy of the center-of-mass motion, $1.5 \hbar \omega$. This ground-state energy is higher than that of three fully polarized fermions, which is,

$$
E_{\mathrm{gs}}^{\uparrow \uparrow}=1.5 \hbar \omega+2.5 \hbar \omega+2.5 \hbar \omega=6.5 \hbar \omega .
$$

Thus, in the presence of repulsive interactions, the ground state of three fully polarized fermions is unstable with respect to a single spin-flip, suggestive of an itinerant ferromagnetic transition at a certain scattering length for three fermions.

\section{QUANTUM VIRIAL EXPANSION FOR THERMODYNAMICS}

The few-particle solutions presented previously can provide information about the high-temperature thermodynamics of many-body systems, through a quantum virial expansion of the grand thermodynamic potential $[6,32]$. In the grand canonical ensemble, the thermodynamic potential is given by

$$
\Omega=-k_{B} T \ln \mathcal{Z},
$$

where $k_{B}$ is the Boltzmann constant and

$$
\mathcal{Z}=\operatorname{Tr} \exp \left[-(\mathcal{H}-\mu \mathcal{N}) / k_{B} T\right]
$$

is the grand partition function. We may rewrite this in terms of the partition function of clusters,

$$
Q_{n}=\operatorname{Tr}_{n}\left[\exp \left(-\mathcal{H} / k_{B} T\right)\right],
$$

where the integer $n$ denotes the number of particles in the cluster and the trace $\operatorname{Tr}_{n}$ is taken over $n$-particle states with a proper symmetry. The partition function of clusters $Q_{n}$ can be calculated using the complete solutions of an $n$-particle system. The grand partition function is then written as

$$
\mathcal{Z}=1+z Q_{1}+z^{2} Q_{2}+\cdots,
$$

where $z=\exp \left(\mu / k_{B} T\right)$ is the fugacity. At high temperatures, it is well-known that the chemical potential $\mu$ diverges to $-\infty$, so the fugacity would be very small, $z \ll 1$. We can then expand the high-temperature thermodynamic potential in powers of the small parameter $z$,

$$
\Omega=-k_{B} T Q_{1}\left[z+b_{2} z^{2}+\cdots+b_{n} z^{n}+\cdots\right],
$$

where $b_{n}$ may be referred to as the $n$th (virial) expansion coefficient. It is readily seen that

$$
\begin{gathered}
b_{2}=\left(Q_{2}-Q_{1}^{2} / 2\right) / Q_{1}, \\
b_{3}=\left(Q_{3}-Q_{1} Q_{2}+Q_{1}^{3} / 3\right) / Q_{1}, \text { etc. }
\end{gathered}
$$

These equations present a general definition of the quantum virial expansion and are applicable to both homogeneous and trapped systems. The determination of the $n$th virial coefficient requires knowledge of up to the $n$-body problem.
In practice, it is convenient to concentrate on the interaction effects only. We thus consider the difference $\Delta b_{n} \equiv b_{n}-b_{n}^{(1)}$ and $\Delta Q_{n} \equiv Q_{n}-Q_{n}^{(1)}$, where the superscript "1" denotes the noninteracting systems. For the second and third virial coefficient, we shall calculate

$$
\Delta b_{2}=\Delta Q_{2} / Q_{1}
$$

and

$$
\Delta b_{3}=\Delta Q_{3} / Q_{1}-\Delta Q_{2} .
$$

\section{A. Noninteracting virial coefficients}

The background noninteracting virial coefficients can be conveniently determined by the noninteracting thermodynamic potential. For a homogeneous two-component Fermi gas, this takes the form

$$
\Omega_{\mathrm{hom}}^{(1)}=-V \frac{2 k_{B} T}{\lambda^{3}} \frac{2}{\sqrt{\pi}} \int_{0}^{\infty} t^{1 / 2} \ln \left(1+z e^{-t}\right) d t,
$$

where $\lambda \equiv\left[2 \pi \hbar^{2} /\left(m k_{B} T\right)\right]^{1 / 2}$ is the thermal wavelength and $Q_{1, \text { hom }}=2 \mathrm{~V} / \lambda^{3}$. This leads to

$$
b_{n, \text { hom }}^{(1)}=\frac{(-1)^{n+1}}{n^{5 / 2}} .
$$

Hereafter, we use the subscript "hom" to denote the quantity in the homogeneous case; otherwise, by default we refer to a trapped system.

For a harmonically trapped Fermi gas, the noninteracting thermodynamic potential in the semiclassical limit (neglecting the discreteness of the energy spectrum) is

$$
\Omega^{(1)}=-\frac{2\left(k_{B} T\right)^{4}}{(\hbar \omega)^{3}} \frac{1}{2} \int_{0}^{\infty} t^{2} \ln \left(1+z e^{-t}\right) d t,
$$

where $Q_{1}=2\left(k_{B} T\right)^{3} /(\hbar \omega)^{3}$. Taylor expanding in powers of $z$ gives rise to

$$
b_{n}^{(1)}=\frac{(-1)^{n+1}}{n^{4}} .
$$

We note that the noninteracting virial coefficients in the homogeneous case and the trapped case are related by

$$
b_{n}^{(1)}=\frac{b_{n, \mathrm{hom}}^{(1)}}{n^{3 / 2}} .
$$

\section{B. Second virial coefficient in a harmonic trap}

We now calculate the second virial coefficient of a trapped interacting Fermi gas. In a harmonic trap, the oscillator length $d$ provides a large length scale, compared to the thermal wavelength $\lambda$. Alternatively, we may use $\tilde{\omega}=\hbar \omega / k_{B} T \ll 1$ to characterize the intrinsic length scale relative to the trap. All the virial coefficients and cluster partition functions in harmonic traps therefore depend on the small parameter $\tilde{\omega}$. We shall be interested in a universal regime with vanishing $\tilde{\omega}$, in accord with the large number of atoms in a real experiment.

To obtain $\Delta b_{2}$, we consider separately $\Delta Q_{2}$ and $Q_{1}$. The single-particle partition function $Q_{1}$ is determined by the single-particle spectrum of a $3 \mathrm{D}$ harmonic oscillator, 
$E_{n l}=(2 n+l+3 / 2) \hbar \omega$. We find that $Q_{1}=2 /[\exp (+\tilde{\omega} / 2)-$ $\exp (-\tilde{\omega} / 2)]^{3} \simeq 2\left(k_{B} T\right)^{3} /(\hbar \omega)^{3}$. The prefactor of 2 accounts for the two possible spin states of a single fermion. In the calculation of $\Delta Q_{2}$, it is easy to see that the summation over the center-of-mass energy gives exactly $Q_{1} / 2$. Using Eq. (10), we find that

$$
\Delta b_{2}^{\mathrm{att}}=\frac{1}{2} \sum_{v_{n}}\left[e^{-\left(2 v_{n}+3 / 2\right) \tilde{\omega}}-e^{-\left(2 v_{n}^{(1)}+3 / 2\right) \tilde{\omega}}\right],
$$

where the noninteracting $v_{n}^{(1)}=n(n=0,1,2, \ldots$,$) and the$ superscript "att" (or "rep") means the coefficient of an attractively (or repulsively) interacting Fermi gas. The second virial coefficient of a trapped attractive Fermi gas in the BEC-BCS crossover was given in Fig. 3(a) of Ref. [6].

To consider the second virial coefficient of a repulsively interacting Fermi gas, we shall restrict ourselves to a positive scattering length and exclude the lowest ground-state energy level in the summation of the first term in Eq. (49), which corresponds to a bound molecule.

\section{Unitarity limit}

At resonance with an infinitely large scattering length, the spectrum is known exactly, $v_{n, \infty}=n-1 / 2$, giving rise to

$$
\Delta b_{2, \infty}^{\mathrm{att}}=\frac{1}{2} \frac{\exp (-\tilde{\omega} / 2)}{[1+\exp (-\tilde{\omega})]}=+\frac{1}{4}-\frac{1}{32} \tilde{\omega}^{2}+\cdots .
$$

For a repulsive Fermi gas in the unitarity limit, we shall discard the lowest "molecular" state with $v_{0, \infty}=-1 / 2$ and, therefore,

$$
\Delta b_{2, \infty}^{\text {rep }}=\frac{1}{2} \frac{\exp (-\tilde{\omega} / 2)}{[1+\exp (+\tilde{\omega})]}=-\frac{1}{4}+\frac{\tilde{\omega}}{4}+\cdots .
$$

The term $\tilde{\omega}^{2}$ or $\tilde{\omega}$ in Eqs. (50) and (51) is nonuniversal and is negligibly small for a cloud with a large number of atoms. We therefore obtain the universal second virial coefficients: $\Delta b_{2, \infty}^{\text {att }}=+1 / 4$ and $\Delta b_{2, \infty}^{\text {rep }}=-1 / 4$, which are temperature independent.

\section{Third virial coefficient in a harmonic trap}

The calculation of the third virial coefficient, which is given by $\Delta b_{3}=\Delta Q_{3} / Q_{1}-\Delta Q_{2}$, is more complicated. Either the term $\Delta Q_{3} / Q_{1}$ or $\Delta Q_{2}$ diverges as $\tilde{\omega} \rightarrow 0$, but the leading divergences cancel with each other. In the numerical calculation, we have to carefully separate the leading divergent terms and calculate them analytically. It is readily seen that the spin states of $\uparrow \downarrow \uparrow$ and $\downarrow \uparrow \downarrow$ configurations contribute equally to $Q_{3}$. The term $Q_{1}$ in the denominators is canceled exactly by the summation over the center-of-mass energy. We thus have

$$
\begin{aligned}
\Delta Q_{3} / Q_{1}= & {\left[\sum \exp \left(-E_{\mathrm{rel}} / k_{B} T\right)\right.} \\
& \left.-\sum \exp \left(-E_{\mathrm{rel}}^{(1)} / k_{B} T\right)\right] .
\end{aligned}
$$

To proceed, it is important to analyze analytically the behavior of $E_{\text {rel }}$ at high energies. For this purpose, we introduce a relative energy $\bar{E}_{\text {rel }}$, which is the solution of Eq. (23) in the absence of the exchange term $C_{n m}$ and can be constructed directly from the two-body relative energy. In the subspace with a total relative momentum $l$, it takes the form

$$
\bar{E}_{\mathrm{rel}}=(2 n+l+3 / 2) \hbar \omega+(2 v+3 / 2) \hbar \omega,
$$

where $v$ is the solution of the two-body spectrum of Eq. (11). At high energies the full spectrum $E_{\text {rel }}$ approaches asymptotically to $\bar{E}_{\text {rel }}$ as the exchange effect becomes increasingly insignificant. There is an important exception, however, occurring at zero total relative momentum $l=0$. As mentioned earlier, the solution of $\bar{E}_{\text {rel }}$ at $n=0$ and $l=0$ is spurious and does not match any solution of $E_{\text {rel }}$. Therefore, for the $l=0$ subspace, we require $n \geqslant 1$ in Eq. (53).

It is easy to see that if we keep the spurious solution in the $l=0$ subspace, the difference $\left[\sum \exp \left(-\bar{E}_{\text {rel }} / k_{B} T\right)-\right.$ $\left.\sum \exp \left(-E_{\mathrm{rel}}^{(1)} / k_{B} T\right)\right]$ is exactly equal to $\Delta Q_{2}$, since in Eq. (53) the first part of the spectrum is exactly identical to the spectrum of the center-of-mass motion. The spurious solution gives a contribution,

$$
\sum_{v_{n}}\left[e^{-\left(2 v_{n}+3\right) \tilde{\omega}}-e^{-\left(2 v_{n}^{(1)}+3\right) \tilde{\omega}}\right] \equiv 2 e^{-3 \tilde{\omega} / 2} \Delta b_{2}^{\text {att }},
$$

which should be subtracted. Keeping this in mind, we finally arrive at the following expression for the third virial coefficient of a trapped Fermi gas with repulsive interactions:

$$
\Delta b_{3}^{\mathrm{att}}=\sum\left[e^{-\frac{E_{\mathrm{rel}}}{k_{B} T}}-e^{-\frac{\bar{E}_{\mathrm{rel}}}{k_{B} T}}\right]-2 e^{-3 \tilde{\omega} / 2} \Delta b_{2}^{\mathrm{att}} .
$$

The summation is over all the possible relative energy levels $E_{\text {rel }}$ and their asymptotic values $\bar{E}_{\text {rel }}$. It is well-behaved and converges at any scattering length. The third virial coefficient of a trapped attractive Fermi gas in the BEC-BCS crossover is given in Fig. 3(b) of Ref. [6].

\section{Unitarity limit}

In the unitarity limit, it is more convenient to use the exact spectrum given by Eq. (27), where $s_{l, n}$ can be obtained numerically to arbitrary accuracy and the noninteracting $s_{l, n}^{(1)}$ is given by Eq. (30). To control the divergence problem, we shall use the same strategy as before and to approach $s_{l, n}$ by using its asymptotic value $\bar{s}_{l, n}$ given in Eq. (31).

Integrating out the $q$ degree of freedom and using Eq. (50) to calculate $\Delta Q_{2}$, we find that

$$
\Delta b_{3, \infty}^{\mathrm{att}}=\frac{e^{-\tilde{\omega}}}{1-e^{-2 \tilde{\omega}}}\left[\sum_{l, n}\left(e^{-\tilde{\omega} s_{l, n}}-e^{-\tilde{\omega} \bar{s}_{l, n}}\right)+A\right],
$$

where $A$ is given by

$$
A=\sum_{l, n}\left(e^{-\tilde{\omega} \bar{s}_{l, n}}-e^{-\tilde{\omega} s_{l, n}^{(1)}}\right)-\frac{e^{-\tilde{\omega}}}{\left(1-e^{-\tilde{\omega}}\right)^{2}} .
$$

We note that, for the summation, implicitly there is a prefactor $(2 l+1)$, accounting for the degeneracy of each subspace. The value of $A$ can then be calculated analytically, leading to

$$
A=-e^{-\tilde{\omega}}\left(1-e^{-\tilde{\omega}}\right) .
$$


We have calculated numerically $\sum_{l, n}\left(e^{-\tilde{\omega} s_{l, n}}-e^{-\tilde{\omega} \bar{s}_{l, n}}\right)$ by imposing the cutoffs of $n<n_{\max }=512$ and $l<l_{\max }=512$. We find that

$$
\Delta b_{3, \infty}^{\mathrm{att}} \simeq-0.06833960+0.038867 \tilde{\omega}^{2}+\cdots .
$$

The numerical accuracy can be further improved by suitably enlarging $n_{\max }$ and $l_{\max }$. For a Fermi gas with infinitely large repulsions, we need to exclude the states involving a molecule. Thus, in the calculation of $\Delta Q_{3} / Q_{1}$, we exclude the energy levels associated with $s_{l, n=0}$, as given by Eq. (32). In the calculation of $\Delta Q_{2}$, we shall remove the lowest two-body state with $v_{0, \infty}=-1 / 2$. In the end, we find that

$$
\Delta b_{3, \infty}^{\text {rep }} \simeq 0.34976-0.77607 \tilde{\omega}+\cdots .
$$

By neglecting the dependence on $\tilde{\omega}$ in the thermodynamic limit, we obtain the universal third virial coefficients:

$$
\begin{gathered}
\Delta b_{3, \infty}^{\text {att }} \simeq-0.06833960, \\
\Delta b_{3, \infty}^{\text {rep }} \simeq 0.34976 .
\end{gathered}
$$

\section{Unitary virial coefficients in homogeneous space}

We have so far studied the virial coefficients in a harmonic trap. In the unitarity limit, there is a simple relation between the trapped and the homogeneous virial coefficient, as inspired by Eq. (48). This stems from the universal temperature independence of all virial coefficients in the unitarity limit. In the thermodynamic limit, let us consider the thermodynamic potential of a harmonic trapped Fermi gas in the localdensity approximation $\Omega=\int d \mathbf{r} \Omega(\mathbf{r})$, where $\Omega(\mathbf{r})$ is the local thermodynamic potential:

$$
\Omega(\mathbf{r}) \propto z(\mathbf{r})+b_{2, \infty, \operatorname{hom}} z^{2}(\mathbf{r})+\cdots .
$$

Here, the local fugacity $z(\mathbf{r})=z \exp \left[-V(\mathbf{r}) / k_{B} T\right]$ is determined by the local chemical potential $\mu(\mathbf{r})=\mu-V(\mathbf{r})$. On spatial integration, it is readily seen that the universal (temperature independent) part of the trapped virial coefficient is

$$
b_{n, \infty}=\frac{b_{n, \infty, \text { hom }}}{n^{3 / 2}} .
$$

We therefore immediately obtain that the homogeneous second virial coefficients in the unitarity limit are

$$
\begin{aligned}
& \Delta b_{2, \infty, \text { hom }}^{\text {att }}=+\frac{1}{\sqrt{2}}, \\
& \Delta b_{2, \infty, \text { hom }}^{\text {rep }}=-\frac{1}{\sqrt{2}},
\end{aligned}
$$

and the homogeneous third virial coefficients are

$$
\begin{gathered}
\Delta b_{3, \infty, \text { hom }}^{\text {att }} \simeq-0.35501298, \\
\Delta b_{3, \infty, \text { hom }}^{\text {rep }} \simeq+1.8174 .
\end{gathered}
$$

The homogeneous virial coefficients are therefore significantly larger than their trapped counterparts. The factor of $n^{3 / 2}$ is clearly due to the higher density of states in a harmonically trapped geometry.

\section{HIGH-T EQUATION OF STATE OF A STRONGLY INTERACTING FERMI GAS}

We are now ready to calculate the equations of state in the high temperature regime, by using the thermodynamic potentials

$$
\Omega_{\mathrm{hom}}=\Omega_{\mathrm{hom}}^{(1)}-V \frac{2 k_{B} T}{\lambda^{3}}\left(\Delta b_{2, \mathrm{hom}} z^{2}+\cdots\right)
$$

and

$$
\Omega=\Omega^{(1)}-\frac{2\left(k_{B} T\right)^{4}}{(\hbar \omega)^{3}}\left(\Delta b_{2} z^{2}+\Delta b_{3} z^{3}+\cdots\right),
$$

respectively, for a homogeneous or a harmonically trapped Fermi gas. Here, the noninteracting thermodynamic potentials are given by Eqs. (44) and (46). All the other thermodynamic quantities can be derived from the thermodynamic potential by the standard thermodynamic relations, for example, $N=$ $-\partial \Omega / \partial \mu, S=-\partial \Omega / \partial T$, and then $E=\Omega+T S+\mu N$.

As a concrete example, let us focus on the unitarity limit in the thermodynamic limit, which is of the greatest interest. The equations of state are easy to calculate because of the temperature independence of virial coefficients. It is also easy to check the well-known scaling relation in the unitarity limit: $E=-3 \Omega / 2$ for a homogeneous Fermi gas [32] and $E=-3 \Omega$ for a harmonically trapped Fermi gas [4]. The difference of the factor of 2 arises from the fact that, according to the virial theorem, in harmonic traps the internal energy is exactly equal to the trapping potential energy.

To be dimensionless, we take the Fermi temperature $T_{F}$ or the Fermi energy $\left(E_{F}=k_{B} T_{F}\right)$ as the units for temperature and energy. For a homogeneous or a harmonically trapped Fermi gas, the Fermi energy is given by $E_{F}=\hbar^{2}\left(3 \pi^{2} N / V\right)^{2 / 3} / 2 m$ and $E_{F}=(3 N)^{1 / 3} \hbar \omega$, respectively. In the actual calculations, we determine the number of atoms $N$, the total entropy $S$, and the total energy $E$ at a given fugacity and a fixed temperature (i.e., $T=1$ ) and consequently obtain the Fermi temperature $T_{F}$ and the Fermi energy $E_{F}$. We then plot the energy or energy per particle, $E /\left(N E_{F}\right)$ and $S /\left(N k_{B}\right)$, as a function of the reduced temperature $T / T_{F}$.

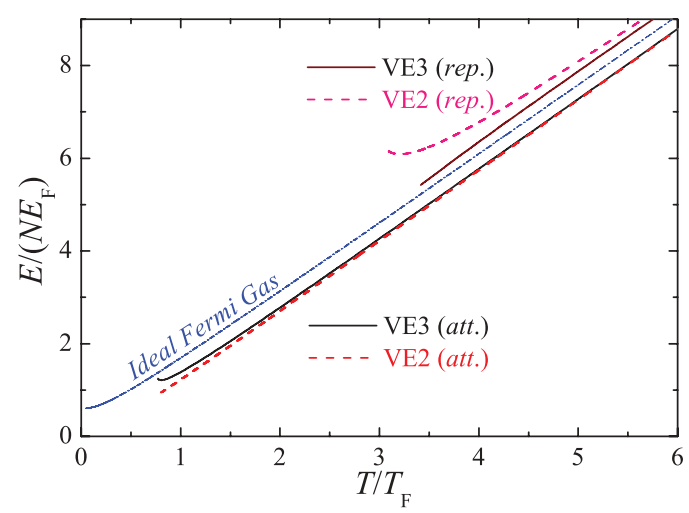

FIG. 4. (Color online) Energy per particle $E /\left(N E_{F}\right)$ as a function of reduced temperature $T / T_{F}$ for a homogeneous Fermi gas with infinitely attractive and repulsive interactions. The predictions of quantum virial expansion up to the second- and third-order are shown by solid lines and dashed lines, respectively. For comparison, we plot the ideal gas result with the dot-dashed line. 


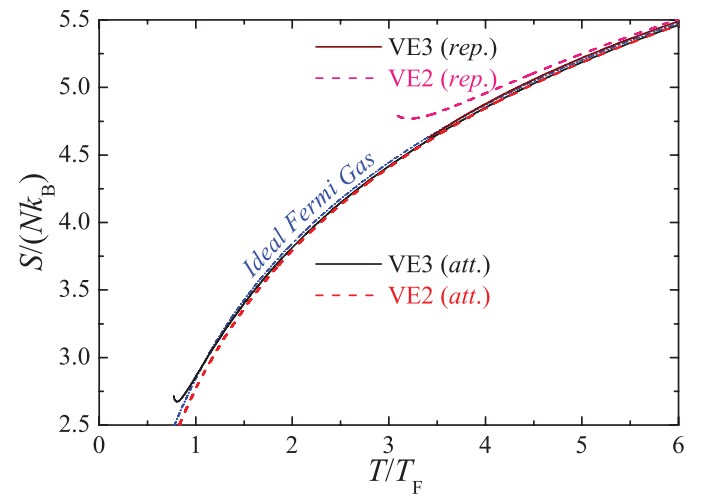

FIG. 5. (Color online) Entropy per particle $S /\left(N E_{F}\right)$ as a function of reduced temperature $T / T_{F}$ for a homogeneous Fermi gas with infinitely attractive and repulsive interactions. The other details are the same as in Fig. 4.

\section{A. Homogeneous equation of state}

We report in Figs. 4 and 5 the temperature dependence of the energy and entropy of a strongly attractively or repulsively interacting homogeneous Fermi gas. The solid line and the dashed line are the predictions of the quantum virial expansion up to the third order (VE3) and the second order (VE2), respectively. For comparison, we also show the ideal gas result by the thin dot-dashed line.

For a strongly attractively interacting Fermi gas, we observe that the quantum virial expansion is valid down to the degeneracy temperature $T_{F}$, where the predictions using the second-order or third-order expansion do not greatly differ. We note that our prediction of the third virial coefficient of a unitarity Fermi gas, $\Delta b_{3, \infty \text {, hom }}^{\text {att }} \simeq-0.35501298$, was experimentally confirmed to within $5 \%$ relative accuracy in the most recent thermodynamic measurement at École Normale Supérieure (ENS) by Nascimbène and co-workers [7].

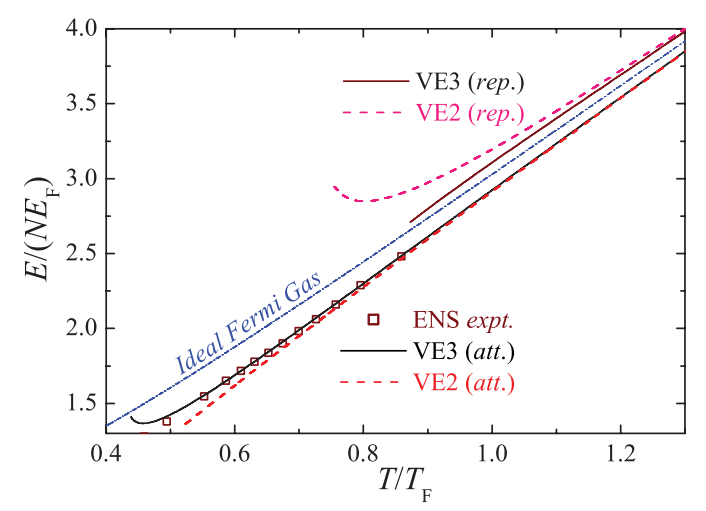

FIG. 6. (Color online) Energy per particle $E /\left(N E_{F}\right)$ as a function of reduced temperature $T / T_{F}$ for a trapped Fermi gas with infinitely attractive and repulsive interactions. The predictions of quantum virial expansion up to the second and third order are shown by solid lines and dashed lines, respectively. For comparison, we plot the ideal gas result with the dot-dashed line. We show also the experimental data measured at ENS by empty squares for an attractive Fermi gas at unitarity $[4,7]$, which agree extremely well with the prediction from quantum virial expansion.

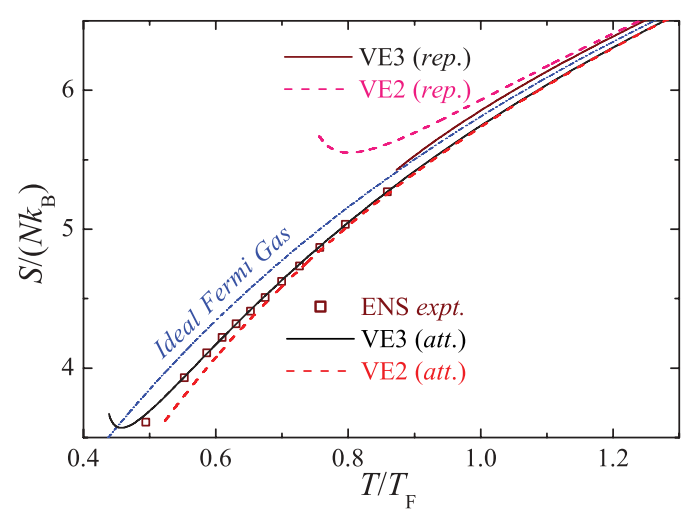

FIG. 7. (Color online) Entropy per particle $S /\left(N E_{F}\right)$ as a function of reduced temperature $T / T_{F}$ for a trapped Fermi gas with infinitely attractive and repulsive interactions. The other details are the same as in Fig. 6.

However, for a strongly repulsively interacting Fermi gas, the applicability of the quantum virial expansion is severely reduced: it seems to be applicable only for $T>5 T_{F}$. Below this characteristic temperature, the difference between the second-order and the third-order prediction becomes very significant. This is partly due to the large absolute value of the third virial coefficient, suggesting that in this case the virial expansion converges very slowly.

\section{B. Harmonically trapped equation of states}

We finally present in Figs. 6 and 7 the high-temperature expansion prediction for the equation of state of a harmonically trapped Fermi gas in the strongly interacting regime. Due to the significantly reduced virial coefficients, the virial expansion in a trap has much broader applicability. For a strongly attractively interacting Fermi gas, it is now quantitatively applicable down to $0.5 T_{F}$, as confirmed by the precise experimental measurement at ENS (empty squares) [4,7]. At the same time, the virial expansion for a strongly repulsively interacting gas seems to be qualitatively valid at $T>T_{F}$. At this temperature, the energy of the repulsively interacting Fermi gas is only marginally higher than the ideal, noninteracting energy. Considering the large energy difference between a fully polarized Fermi gas and a nonpolarized Fermi gas (i.e., at the order of $N E_{F}$ ), we conjecture that a strongly repulsively interacting Fermi gas does not have itinerant ferromagnetism in the temperature regime where the quantum virial expansion theory is applicable.

\section{CONCLUSIONS AND OUTLOOK}

In conclusion, we have presented a complete set of exact solutions for three attractively interacting fermions in a harmonic trap, with either positive or negative scattering lengths. First, we have outlined the details of our previous studies on the quantum virial expansion [6], in particular the method for calculating the third virial coefficient which was recently confirmed experimentally. In addition, we have opened up the previously unexplored repulsively interacting regime and have presented a few-body perspective of itinerant ferromagnetism. We have also studied the high-temperature 
thermodynamics of a strongly repulsively interacting Fermi gas, by calculating its second and third virial coefficients in the unitarity limit.

On the positive scattering length side of a Feshbach resonance, a repulsively interacting Fermi gas is thought to occur by excluding all the many-body states which contain a moleculelike bound state for any two atoms with unlike spins. Strictly speaking, this is a conjecture which stems from a two-body picture. We have examined this conjecture using the exact three-fermion energy spectrum near the resonance. We have found some horizontal energy levels that may be identified as the energy spectrum of three repulsively interacting fermions, as well as some vertical energy levels involving a tightly bound molecule. However, many avoided crossings between horizontal and vertical levels make it difficult to unambiguously identify the energy spectrum of a repulsive Fermi system.

For three repulsively interacting fermions in a harmonic trap, we have shown that close to the resonance the groundstate energy is higher than that of three fully polarized fermions. This is an indication of the existence of itinerant ferromagnetism in a trapped strongly repulsively interacting Fermi gas. We have also considered the possibility of itinerant ferromagnetism at high temperatures. We have found that it does not exist in the regime where a quantum virial expansion is applicable. This gives an upper bound $\left(\sim T_{F}\right)$ for the critical ferromagnetic transition temperature.

Our high-temperature equations of state of a strongly repulsively interacting Fermi gas have a number of potential applications. We anticipate that these results can provide an unbiased benchmark for future quantum Monte Carlo simulations of strongly repulsively interacting Fermi gases at high temperatures [33-35], using either hard-sphere interatomic potentials or resonance interactions. These results are also directly testable in future experimental measurements, as inspired by the most recent thermodynamics measurements at ENS that have already confirmed our predicted second and third virial coefficients for strongly attractively interacting fermions [7]. Our exact three-fermion solutions in 3D harmonic traps will also be useful for understanding the dynamical properties of strongly interacting Fermi gases at high temperatures by applying a similar quantum virial expansion for the dynamic structure factors [36] and single-particle spectral functions [37].

These exact solutions of three interacting particles can be generalized to other dimensions, by adopting a suitable Bethe-Peierls boundary condition for the contact interactions. Of particular interest is the case of two dimensions, where the reduction of the spatial dimensionality increases the role of fluctuations and therefore imposes severe challenges for theoretical studies. The three-body solutions in 2D and the resulting high-temperature equations of state of strongly interacting systems will be given elsewhere and provide a useful starting point to understanding more sophisticated collective phenomena such as the Berezinsky-Kosterlitz-Thouless transition and non-Fermi-liquid behavior.

Note added. Recently, we became aware of a very recent work by Daily and Blume [38], in which the energy spectrum of three and four fermions has been calculated using hyperspherical coordinates with a stochastic variational appoach.
Our exact results are in excellent agreement with theirs when there is an overlap.

\section{ACKNOWLEDGMENTS}

This work was supported in part by the ARC Centre of Excellence, ARC Discovery Projects No. DP0984522 and No. DP0984637, NSFC Grant No. 10774190, and NFRPC (Chinese 973) Grants No. 2006CB921404 and No. 2006CB921306.

\section{APPENDIX A: CALCULATION OF $\boldsymbol{C}_{n n^{\prime}}$}

In this appendix, we outline the details of how to construct the matrix element $C_{n n^{\prime}}$ in Eq. (23), which is given by

$$
C_{n n^{\prime}} \equiv \int_{0}^{\infty} \rho^{2} d \rho R_{n l}(\rho) R_{n^{\prime} l}\left(\frac{\rho}{2}\right) \psi_{2 b}^{\mathrm{rel}}\left(\frac{\sqrt{3}}{2} \rho ; v_{l, n^{\prime}}\right)
$$

where

$$
R_{n l}(\rho)=\sqrt{\frac{2 n !}{\Gamma(n+l+3 / 2)}} \rho^{l} e^{-\rho^{2} / 2} L_{n}^{(l+1 / 2)}\left(\rho^{2}\right)
$$

is the radial wave function of an isotropic 3D harmonic oscillator and the two-body relative wave function is

$$
\psi_{2 b}^{\text {rel }}=\Gamma\left(-v_{l, n^{\prime}}\right) U\left(-v_{l, n^{\prime}}, \frac{3}{2}, \frac{3}{4} \rho^{2}\right) \exp \left(-\frac{3}{8} \rho^{2}\right) .
$$

Here, for convenience we have set $d=1$ as the unit of length. $L_{n}^{(l+1 / 2)}$ is the generalized Laguerre polynomial and $U$ is the second Kummer confluent hypergeometric function. A direct integration for $C_{n n^{\prime}}$ is difficult, since the second Kummer function has a singularity at the origin. The need to integrate for different values of $v_{l, n^{\prime}}$ also causes additional complications.

It turns out that a better strategy for the numerical calculations is to write

$$
\psi_{2 b}^{\mathrm{rel}}=\sum_{k=0}^{\infty} \frac{1}{k-v_{l, n^{\prime}}} \sqrt{\frac{\Gamma(k+3 / 2)}{2 k !}} R_{k 0}\left(\frac{\sqrt{3}}{2} \rho\right),
$$

by using the exact identity

$$
\Gamma(-v) U\left(-v, \frac{3}{2}, x^{2}\right)=\sum_{k=0}^{\infty} \frac{L_{k}^{1 / 2}\left(x^{2}\right)}{k-v} .
$$

Therefore, we find that

$$
C_{n n^{\prime}}=\sum_{k=0}^{\infty} \frac{1}{k-v_{l, n^{\prime}}} \sqrt{\frac{\Gamma(k+3 / 2)}{2 k !}} C_{n n^{\prime} k}^{l},
$$

where

$$
C_{n n^{\prime} k}^{l} \equiv \int_{0}^{\infty} \rho^{2} d \rho R_{n l}(\rho) R_{n^{\prime} l}\left(\frac{\rho}{2}\right) R_{k 0}\left(\frac{\sqrt{3}}{2} \rho\right)
$$

can be calculated to high accuracy with an appropriate integration algorithm. In checking convergence of the summation over $k$, we find numerically that for a cutoff $n_{\max }$ (i.e., $n, n^{\prime}<n_{\max }$ ), $C_{n n^{\prime} k}^{l}$ vanishes for a sufficiently large $k>k_{\max } \sim 4 n_{\max }$.

In practical calculations, we tabulate $C_{n n^{\prime} k}^{l}$ for a given total relative angular momentum. The calculation of $C_{n n^{\prime}}$ for 
different values of $v_{l, n^{\prime}}$ then reduces to a simple summation over $k$, which is very efficient. Numerically, we have confirmed that the matrix $C_{n n^{\prime}}$ is symmetric, that is, $C_{n n^{\prime}}=$ $C_{n^{\prime} n}$.

\section{APPENDIX B: CALCULATION OF $s_{l, n}$}

The calculation of $s_{l, n}$ seems straightforward by using the Bethe-Peierls boundary condition in hyperspherical coordinates (28). However, we find that numerical accuracy is low for large $n$ and $l$ due to the difficulty of calculating the hypergeometric function ${ }_{2} F_{1}$ accurately using IEEE standard precision arithmetic. We have therefore utilized MATHEMATICA software that can perform analytical calculations with unlimited accuracy. For this purpose, we introduce
$\Delta s_{l, n}=s_{l, n}-\bar{s}_{l, n}$. After some algebra, we find the following boundary condition for $t \equiv \Delta s_{l, n} / 2$,

$$
\sin (\pi t)=\sqrt{\frac{\pi}{3}} \frac{(-1)^{n+l} \Gamma(n+l+1+t)}{2^{l} \Gamma\left(l+\frac{3}{2}\right) \Gamma(n+1+t)} f(t),
$$

where we have defined a function

$$
f(t) \equiv{ }_{2} F_{1}\left(-n-t, n+l+1+t, l+\frac{3}{2} ; \frac{1}{4}\right) .
$$

This equation can be solved using the MATHEMATICA routine "FindRoot," by seeking a solution around $t=0$. It is also easy to write a short program to solve Eq. (B1) continuously for $n<n_{\max }=512$ and $l<l_{\max }=512$. In a typical current PC, this takes several days. The results can be tabulated and stored in a file for further use.
[1] E. Braaten and H. Hammer, Phys. Rep. 428, 259 (2006).

[2] I. Bloch, J. Dalibard, and W. Zwerger, Rev. Mod. Phys. 80, 885 (2008).

[3] S. Giorgini, L. P. Pitaevskii, and S. Stringari, Rev. Mod. Phys. 80, 1215 (2008).

[4] H. Hu, X.-J. Liu, and P. D. Drummond, New J. Phys. 12, 063038 (2010).

[5] C. Chin, R. Grimm, P. Julienne, and E. Tiesinga, Rev. Mod. Phys. 82, 1225 (2010).

[6] X.-J. Liu, H. Hu, and P. D. Drummond, Phys. Rev. Lett. 102, 160401 (2009).

[7] S. Nascimbène, N. Navon, K. Jiang, F. Chevy, and C. Salomon, Nature (London) 463, 1057 (2010).

[8] G.-B. Jo, Y.-R. Lee, J.-H. Choi, C. A. Christensen, T. H. Kim, J. H. Thywissen, D. E. Pritchard, and W. Ketterle, Science 325, 1521 (2009).

[9] D. Blume and C. H. Greene, Phys. Rev. A 66, 013601 (2002).

[10] J. von Stecher and C. H. Greene, Phys. Rev. Lett. 99, 090402 (2007).

[11] D. Blume and K. M. Daily, Phys. Rev. A 80, 053626 (2009).

[12] D. S. Petrov, C. Salomon, and G. V. Shlyapnikov, Phys. Rev. Lett. 93, 090404 (2004).

[13] For a brief review, see for example, F. Ferlaino and R. Grimm, Physics 3, 9 (2010).

[14] E. C. Stoner, Proc. R. Soc. London A 165, 372 (1938).

[15] R. A. Duine and A. H. MacDonald, Phys. Rev. Lett. 95, 230403 (2005).

[16] S. Zhang, H.-H. Hung, and C. Wu, e-print arXiv:0805.3031.

[17] L. J. LeBlanc, J. H. Thywissen, A. A. Burkov, and A. Paramekanti, Phys. Rev. A 80, 013607 (2009).

[18] G. J. Conduit and B. D. Simons, Phys. Rev. Lett. 103, 200403 (2009).
[19] G. J. Conduit, A. G. Green, and B. D. Simons, Phys. Rev. Lett. 103, 207201 (2009).

[20] H. Zhai, Phys. Rev. A 80, 051605(R) (2009).

[21] X. Cui and H. Zhai, Phys. Rev. A 81, 041602(R) (2010).

[22] S. Pilati, G. Bertaina, S. Giorgini, and M. Troyer, Phys. Rev. Lett. 105, 030405 (2010).

[23] S.-Y. Chang, M. Randeria, and N. Trivedi, e-print arXiv:1004.2680.

[24] H. Dong, H. Hu, X.-J. Liu, and P. D. Drummond, Phys. Rev. A 82, 013627 (2010).

[25] T.-L. Ho, Phys. Rev. Lett. 92, 090402 (2004).

[26] H. Hu, P. D. Drummond, and X.-J. Liu, Nat. Phys. 3, 469 (2007).

[27] T. Busch, B. G. Englert, K. Rzazewski, and M. Wilkens, Found. Phys. 28, 549 (1998).

[28] F. Werner and Y. Castin, Phys. Rev. Lett. 97, 150401 (2006).

[29] F. Werner and Y. Castin, Phys. Rev. A 74, 053604 (2006).

[30] F. Werner, Ph.D. thesis, École Normale Supérieure, 2008.

[31] J. P. Kestner and L.-M. Duan, Phys. Rev. A 76, 033611 (2007).

[32] T.-L. Ho and E. J. Mueller, Phys. Rev. Lett. 92, 160404 (2004)

[33] V. K. Akkineni, D. M. Ceperley, and N. Trivedi, Phys. Rev. B 76, 165116 (2007).

[34] A. Bulgac, J. E. Drut, and P. Magierski, Phys. Rev. Lett. 96, 090404 (2006).

[35] E. Burovski, N. Prokof'ev, B. Svistunov, and M. Troyer, Phys. Rev. Lett. 96, 160402 (2006).

[36] H. Hu, X.-J. Liu, and P. D. Drummond, Phys. Rev. A 81, 033630 (2010).

[37] H. Hu, X.-J. Liu, P. D. Drummond, H. Dong, Phys. Rev. Lett. 104, 240407 (2010).

[38] K. M. Daily and D. Blume, Phys. Rev. A 81, 053615 (2010). 